\section{Die neue Spezialitätenliste (SL): Einführungstermin 1. Juli 2001 gilt auch für die Ärzteschaft}

\author{
Max Giger, Mitglied Zentralvorstand FMH, Winterthur
}

Auf den 1. Juli 2001 hat das Bundesamt für Sozialversicherung (BSV) die neue SL mit den ab diesem Datum gültigen Höchstpreisen für pharmazeutische Spezialitäten und konfektionierte Arzneimittel, die Pflichtleistungen für die Krankenversicherer sind, in Kraft gesetzt. Die Publikation erfolgte auf der Homepage des BSV (http://www.bsv.admin.ch/sl/liste/d/ index.htm) und auf einer CD-ROM, die an alle Leistungserbringer versandt wurde. Die gedruckte SL wird auf zweite Hälfte August 2001 in Aussicht gestellt. Das BSV wurde durch die FMH darauf hingewiesen, dass dadurch Abgabestellen, die weder über Internetzugang noch über CD-ROM-Laufwerk verfügen, vor logistische Probleme gestellt werden.

Es muss hier festgehalten werden, und dies entgegen leider wiederholt verbreiteter Irrmeinungen, dass die neuen SL-Preise seit dem 1. Juli 2001 für alle verbindlich sind. So müssen alle seit dem 1. Juli abgegebenen, auf der SL aufgeführten Arzneimittel mit dem neuen Preis verrechnet werden. Es besteht hier keine sogenannte Ausverkaufsfrist nach altem Preis.

Seit Januar 2001 wurden keine Preise mehr auf den abgegebenen Arzneimitteln angebracht. Dies ist aus Konsumenten- und Patientensicht eine höchst unbefriedigende Situation. Anlässlich der vom Eidgenössischen Departement des Innern (EDI) einberufenen Tagung zu den Medikamentenkosten vom 2. Juli wurde festgehalten, dass künftig im Interesse des Kostenbewusstseins und der Therapietreue (Compliance) die Abgabepreise der auf der SL aufgeführten Arzneimittel wieder auf den Packungen aufgeführt sein sollen. So empfehlen wir dringend bei der Abgabe von Arzneimitteln, den Preis (Endpreis nach Abzug der nach KVG weiterzugebenden Vergünstigungen, maximal SL-Preis) auf der Packung anzubringen.

Durch die neue Preisgestaltung, die ohne Rücksicht auf die Mischtarife in Kraft gesetzt wurde, entstehen in den Kantonen mit direkter Medikamentenabgabe Einkommensverluste. Die vom Zentralvorstand eingesetzte Verhandlungsdelegation "LOA" führt im Hinblick auf eine Kompensation dieser Verluste mit den Krankenversicherern Verhandlungen zur entsprechenden Anpassung der kantonalen Tarife. Die Resultate werden allen Betroffenen umgehend mitgeteilt werden.

A ce propos, il convient de souligner que, contrairement à une fausse idée largement répandue, les nouveaux prix de la LS sont contraignants pour tout le monde dès le $1^{\text {er }}$ juillet 2001. Depuis lors, tous les médicaments figurant dans cette liste doivent être facturés selon les nouveaux prix. Il n'y a donc pas de délai de liquidation des stocks d'après l'ancien prix.

Depuis le mois de janvier 2001, les prix ne sont plus indiqués sur les produits pharmaceutiques. Cette situation n'est pas du tout satisfaisante pour les patients et pour les consommateurs. Lors de la conférence du 2 juillet sur la maîtrise des coûts des médicaments, organisée par le Département fédéral de l'intérieur (DFI), il a été souligné que, pour susciter une prise de conscience des coûts et améliorer la "compliance", il fallait à nouveau faire figurer le prix de vente des médicaments (prix après déduction des rabais à répercuter conformément à la LAMal; prix maximal fixé par la LS) sur l'emballage. C'est donc ce que nous recommandons instamment.

A cause de ces nouveaux prix qui ont été fixés sans tenir compte des tarifs mixtes, les médecins des cantons où l'on pratique la propharmacie subiront des pertes de revenu. La délégation «RBP» (rémunération basée sur les prestations), instituée par le Comité central de la FMH, a engagé des négociations avec les assureurs-maladie pour adapter les tarifs cantonaux afin de compenser lesdites pertes. Les résultats seront immédiatement communiqués à toutes les personnes concernées. 